\title{
Le clonage du locus $T$ de la souris : une mutation du développement connue depuis longtemps entre dans l'âge de la biologie moléculaire
}

Il y a longtemps que la souris est reconnue comme un modèle de choix pour l'étude de la biologie du développement. Ce constat est devenu encore plus net depuis la mise en œuvre, durant la dernière décennie, de méthodes de plus en plus sophistiquées de manipulation de l'embryon de souris. Cependant notre compréhension du développement de ce mammifère a pris du retard par rapport à celle de deux autres systèmes majeurs d'étude du développement : la drosophile et le nématode. Deux raisons principales sont à l'origine de cette différence : l'une tient à la facilité, pour un expérimentateur même isolé, d'obtenir des mutations à un locus ou dans une région fonctionnelle donnée chez ces deux organismes; l'autre à la relative facilité de cloner l'ADN des régions correspondantes.

La situation est très différente chez la souris: en effet, d'un côté de nombreuses mutations éventuellement intéressantes pour l'étude du développement ont certes été isolées au long des cinquante dernières années, mais elles n'ont pas été clonées; d'un autre côté et symétriquement de nombreux loci dont l'expression est contrôlée au cours du développement ont été clonés, mais on n'en connaît pas de variants mutants.

Ce n'est que dans un très petit nombre de cas que l'ADN d'un locus a été cloné et que le phénotype mutant est connu. Or ce n'est que la combinaison de ces deux outils que peut du mécanisme d'action d'un gène donné dans le processus du développement.

Comment s'y prendre pour obtenir le chaînon manquant dans les cas décrits ci-dessus? En réalité deux problèmes se posent dont les solutions sont différentes. La méthodologie naissante de la recombinaison homologue devrait permettre d'obtenir des mutations à un locus donné, pour peu qu'il soit cloné : le remplacement du gène endogène normal par un gène muté s'effectue sur des cellules maintenues en culture et qui ont la capacité, après injection dans des embryons, de participer à la formation d'un organisme mosaïque et éventuellement à sa lignée germinale $\left(\mathrm{m} / \mathrm{s} n^{\circ} 1\right.$, vol. 6, p. 18) [1]. Bien qu'encore dans son enfance, cette approche est très prometteuse.

L'autre situation est celle où l'on dispose d'un phénotype mutant et où l'on veut cloner le gène correspondant sans avoir la moindre idée sur la nature de son produit ou sur sa fonction précise. Cette démarche est communément appelée la génétique " inverse " (m/s $n^{\circ} 3$, vol. 4, p. 138, 141 et 151) [2] et repose sur l'idée qu'il devrait être possible, en raffinant au maximum les techniques de la génétique classique, de localiser la mutation dans une région précise du génome dont on pourrait ensuite cloner l'ADN grâce à une " marche " sur le chromosome à partir d'un ADN cloné au voisinage de la mutation. Plusieurs moyens s'offrent pour l'identification du gène dont la muta- tion est à l'origine du phénotype observé : hybridation avec l'ARN des tissus appropriés, mise en évidence d'une séquence conservée à travers l'évolution, ou repérage de sites de coupure par des enzymes de restriction connus pour se trouver à l'extrémité 5' de nombreux gènes de mammifères [3]. Malgré certains succès remarquables de la génétique inverse chez les mammifères $\left(\mathrm{m} / \mathrm{s} n^{\circ} 3\right.$, vol. 4 , p. $141 ; n^{\circ} 8$, vol. 5 , p. $589 ; n^{\circ} 3$, vol. 6, p. 292), cette démarche s'est en général avérée beaucoup plus difficile à mettre en pratique que ne le prévoyait la théorie. La difficulté est en général due au fait qu'en dépit d'une localisation génétique précise, celle-ci laisse néanmoins persister une incertitude qui, sur le plan moléculaire, peut se mesurer parfois en centaines de kilobases ou plus : dans ces conditions, retrouver le gène impliqué dans le phénotype et le distinguer des nombreux autres gènes de la région peut s'avérer aussi difficile que rechercher une aiguille dans une botte de foin.

Dans ce contexte, la publication récente par l'équipe de Bernard Herrmann et Hans Lehrach du clonage du gène $T$ apparaît comme particulièrement remarquable [4]. La mutation $\mathrm{T}$ originale fut découverte en 1927 par DobrovolskaïaZavadskaïa alors qu'elle travaillait à l'Institut du radium de Paris [5]. C'est l'une des premières mutations létales à avoir été découverte chez les mammiferes. Ce qui permit au départ de la repérer tenait à un phé- 
notype dominant de raccourcissement de la queue observé chez les hétérozygotes et surajouté à l'effet récessif sur la viabilité des embryons homozygotes.

Comme on peut s'en rendre compte à la lecture des comptes rendus de séances de la société de biologie par Dobrovolskaïa-Zavadskaïa où fut décrite la mutation, la relation entre gènes et développement commençait tout juste à être établie: "Nous croyons opportun de désigner par l'expression caractère (facteur) "non viable" la mortification de la queue après un développement défectueux. Cela signifie que le gène (ou les gènes) correspondant à cet organe existait dans la matière héréditaire de l'animal, que ce gène a déterminé le développement de cet appendice, mais qu'il lui manquait quelque chose pour assurer la vie ultérieure et le développement de l'organe." Bien sûr, depuis cette date, le contrôle génétique du développement a été établi et l'étonnement n'a fait que grandir devant la complexité des activités géniques probablement impliquées dans l'embryogenèse.

Bien que de nombreuses autres mutations du développement aient été décrites depuis une soixantaine d'années, le locus $\mathrm{T}$ a fait l'objet d'une attention particulière de la part des généticiens du développement : c'est pourquoi ses manifestations biologiques sont très bien décrites. Le gène $T$ code pour un produit qui est essentiel dans la formation du mésoderme et la morphogenèse subséquente de la notochorde; les fœtus porteurs de la mutation $\mathrm{T}$ à l'état homozygote meurent vers le $10^{\mathrm{e}}$ jour, c'est-à-dire aux alentours du milieu de la gestation. L'intérêt porté au locus $\mathrm{T}$ a entraîné la recherche et l'isolement de nombreuses autres mutations, et c'est grâce à celles-ci que Herrmann et Lehrach ont pu démontrer sans ambiguité que le gène qu'ils ont cloné et qui s'exprime durant l'embryogenèse est bien le gène $T$.

L'histoire de ce clonage a commencé il y a six ans, lorsque ces auteurs réussirent à microdisséquer la section $\mathrm{m} / \mathrm{s} n^{\circ} 6$ vol. 6 , juin 90 proximale du chromosome 17 à partir d'étalements métaphasiques ; ils purent alors cloner une série de séquences anonymes d'ADN qui servirent par la suite à identifier des marqueurs génétiques de cette région du génome [6, 7]. La ségrégation de ces marqueurs lors d'un croisement en retour à haute résolution avec des animaux hétérozygotes pour le locus $\mathrm{T}$ révéla une liaison très étroite entre ce dernier et un locus défini par l'un des clones anonymes (D17 Leh 119 II). Ce marqueur servit de point de départ à une marche et à des sauts le long du chromosome vers le locus T. A ce stade, l'existence de cinq délétions indépendantes et d'une duplication dans la région du locus $\mathrm{T}$ permettait de le localiser dans une région de $110 \mathrm{~kb}$ distante de $350 \mathrm{~kb}$ du point de départ de la marche. Cette région fut utilisée pour la recherche de séquences qui seraient exprimées chez des embryons âgés de 8,5 jours et une unité de transcription unique fut ainsi identifiée. Si l'histoire s'était arrêtée là, Herrmann aurait simplement disposé d'un bon candidat pour le gène $T$. La preuve que cette unité de transcription et le gène $T$ ne faisaient qu'un fut apportée grâce à l'existence d'une autre mutation $\mathrm{T}$ : en effet l'analyse moléculaire de cette dernière révéla la présence d'un élément de structure rétrovirale qui entraîne de manière spécifique l'interruption de l'unité de transcription mentionnée ci-dessus et empêche ainsi l'expression du produit génique normal [4].

Comme attendu, le produit du gène $T$ est exprimé de manière hautement spécifique durant l'embryogenèse. Des expériences d'hybridation in situ permettent de localiser son ARN messager aussi bien dans le mésoderme précoce que dans l'épithélium dont il est issu; par la suite, l'expression est limitée à la notochorde [8]. Un tel profil d'expression se superpose exactement aux tissus affectés chez les embryons mutants. Le gène $T$ est hautement conservé chez toutes les espèces de vertébrés, ce qui semble confirmer qu'il joue bien un rôle déterminant dans le développement des mammifères. Malheureusement la séquence de ce gène ne ressemble à aucune autre connue et ne fournit donc pas d'éléments sur le rôle biochimique de la protéine. Il faudra donc attendre des analyses supplémentaires pour comprendre sa fonction.

Il y a soixante ans, la découverte par hasard d'une souris ayant une queue plus courte que ses congénères a joué un rôle crucial dans l'émergence d'un nouveau champ de recherche combinant la génétique et la biologie du développement et que l'on peut qualifier de génétique du développement. Le clonage de la mutation à l'origine de la queue courte fait incontestablement sortir ce domaine de l'enfance. Sommes-nous sur le point de découvrir les secrets qui se cachent dans l'embryon? Seul le temps le dira.

Lee M. Silver
Princeton University, Princeton, NJ,
USA.
Charles Babinet
Institut Pasteur, unité de génétique des
mammiferes, 25, rue du Docteur-Roux,
75015 Paris, France.

\section{RÉFÉRENCES}

1. Capecchi M. The new mouse genetics Altering the genome by gene targeting. Trends Genet 1989 ; 5 : 70-6.

2. Orkin SH. Reverse genetics and human disease. Cell $1986 ; 47$ : 845-50.

3. Lindsay S, Brid AP. Use of restriction enzymes to detect potential gene sequences in mammalian DNA. Nature $1987 ; 327$ : 336-8.

4. Herrmann BG, Labeit $\mathrm{S}$, et al. Cloning of the $\mathrm{T}$ gene required in mesoderm formation in the mouse. Nature $1990 ; 343$ : 617-22.

5. Dobrovolskaïa-Zavadskaïa N. Sur la mortification spontanée de la queue chez la souris nouveau-née et sur l'existence d'un caractère (facteur) héréditaire " non viable ". $C R$ Séanc Soc Biol 1927; 97 : 114-6.

6. Röhme D, Fox H, et al. Molecular clones of the mouse $\mathrm{T}$ complex derived from microdissected metaphase chromosomes. Cell 1984 ; 36 : 783-8.

7. Fox H, Marin GR, et al. Molecular probes define different regions of the mouse $t$ complex. Cell 1985 ; 40 : 63-9.

8. Wilkinson DG, Bhatt $\mathrm{S}$, et al. Expression pattern of the mouse $\mathrm{T}$ gene and its role in mesoderm formation. Nature 1990 ; 343 : 657-9. 